\title{
CONSTRUIRE SON IDENTITÉ DANS UN ENVIRONNEMENT MÉDIATIQUE
}

\author{
Yves Patte ${ }^{1}$
}

Cet article est le fruit d'un travail d'enquête qualitative en sociologie auprès d'adolescents et d'un travail ethnographique dans une école secondaire. Le but était de comprendre comment les adolescents, aujourd'hui, utilisaient les médias dans leur travail de construction identitaire. Nous avons pu dégager trois compétences spécifiques, c'est-à-dire trois manières dont ces jeunes utilisent les médias pour se construire individuellement et socialement dans leur groupe de pairs : l'identification, l'affirmation et la mise en scène.

En abordant la question du rôle des médias dans la construction identitaire des jeunes, nous nous intéressons à la manière dont l'individu se construit dans l'environnement médiatique contemporain, et par conséquent à la manière dont ces médias s'intègrent jusqu'au cœur même de la construction de l'individu.

Comment se construire individuellement et socialement dans un univers médiatique omniprésent? Quelles ressources les adolescents

1 Sociologue et enseignant dans le secondaire, consultant pour «Recherche sociale» (http://www.recherchesociale.be/).

Recherches en communication, $\mathrm{n}^{\circ} 33$ (2010). 
peuvent-ils trouver dans les médias pour leur travail de construction identitaire ? Et quelles compétences doivent-ils acquérir et développer pour y arriver?

\section{Cadre théorique et méthodologique}

C'est la manière dont nous abordons la « construction identitaire », et donc le concept d' "identité », qui va définir à la fois notre cadre théorique et notre approche méthodologique.

Nous aborderons donc la construction identitaire à partir de l'idée de " gestion relationnelle de soi », que Bajoit définit comme "le processus par lequel l'individu est sujet, c'est-à-dire gère les tensions existentielles entre les attentes des autres vis-à-vis de lui et ses propres attentes envers lui-même »(Bajoit, 1997, p. 117). Les institutions proposent et imposent à chaque individu une idée de son accomplissement personnel, à laquelle il adhère plus ou moins, c'est-à-dire son identité imaginée ; mais qui ne coïncide jamais totalement avec ce qu'elles attendent de lui, ce qu'elles lui permettent d'être, c'est-à-dire son identité assignée ; ni avec ce qu'il parvient à réaliser concrètement, étant donné les engagements qu'il prend avec lui-même, c'est-à-dire son identité engagée.

La gestion relationnelle de soi est alors le travail auquel se livre l'individu pour faire tenir ensemble ces trois formes d'identité, pour gérer les tensions entre celles-ci. Le résultat de ce travail sera son identité personnelle, le « Je », constamment en évolution et tout en restant toujours la même, ce qu'Erikson (1968) appelle la « continuité de soi ».

Cette approche de l'identité est, comme on le voit, tout à fait relationnelle. Elle s'inscrit dans un cadre social. Nous rejoignons donc Lipiansky (1992) lorsqu'il dit que l'expérience groupale est le lieu privilégié pour aborder la question de l'identité. Cette expérience, dit-il, stimule fortement la conscience de soi. S'instaure une situation d'interaction entre l'individu et le groupe pris dans son ensemble. Se sentant observés, les individus sont davantage réflexifs. Le regard de l'autre prend alors une place essentielle. Et sans que l'enquêteur ait à demander à l'individu de s'expliquer, le discours sur soi est simplement stimulé par la situation de groupe.

Stephanie : En groupe, comme on est entre amis, on n'a pas toujours envie de se payer la gêne, donc on dit pas tout... 
Céline : Ouais, on peut se dire : si je dis un truc débile... les autres vont dire que je suis conne et tout... et on n'a pas envie que ça se passe, quoi...

Cette approche de l'identité par le groupe s'inscrit pleinement dans les recherches sur la réception des produits médiatiques. Pasquier comme Tisseron mettent l'accent sur le fait que lorsqu'on parle d'un film ou d'une série, on parle de soi. Katz et Liebes (1990) avaient montré, à propos de la série "Dallas ", que cette série était utilisée comme support dans les interactions familiales et les relations de voisinage. Parler de la série est ainsi un moyen de faire passer des messages personnels à partir d'un sujet extérieur à soi (Pasquier, 1994). Plus encore, la relation à un programme se joue sur deux registres à la fois : « comme mode de consolidation du soi et comme mode d'affirmation pour les autres » (Pasquier, 1995, p. 37). A travers le texte médiatique, le spectateur formule et élabore divers aspects de son identité personnelle (Liebes, 1994).

$\mathrm{Vu}$ l'importance du groupe de pairs dans le processus de construction identitaire, la recherche a donc été menée auprès de groupes existants, c'est-à-dire déjà constitués, de jeunes qui se connaissent et se côtoient régulièrement. Créer un groupe expérimental pour le but de la recherche aurait effectivement créé un artefact, consistant « à proposer l'ethnographie d'une communauté constituée sur invitations » (Dayan, 1992, p. 196). Concrètement, la recherche a été menée dans différentes écoles secondaires, avec des groupes d'élèves de $5^{\text {ème }}$ année, entre 2001 et 2002. Après une première phase de discussions en groupes, nous avons proposé à celles et ceux qui le souhaitaient des entretiens individuels, ce qui nous a permis de saisir ce qui se jouait spécifiquement dans la situation de groupe, au niveau de la construction sociale de l'identité. Les conclusions ont été étayées par un travail ethnographique dans l'enseignement, entre 2007 et 2010.

Quant aux produits médiatiques discutés lors des entretiens de groupe, le choix s'est fait par les jeunes eux-mêmes, afin d'éviter un autre artefact décrit par Dayan (1992), celui de l'artefact textuel, privilégiant un programme donné, arbitrairement, indépendamment des préoccupations et choix médiatiques des jeunes concernés. C'est donc spontanément que ces jeunes nous ont parlé majoritairement des séries télévisées et, dans une moindre mesure, des reality-shows. 
Nous avons donc essayé d'intégrer le moins d'artefacts possibles dans le travail empirique et de reproduire au maximum la situation de groupe ordinaire lors des récréations dans les écoles.

\section{Premier constat}

Un premier constat, tout d'abord, très bref, qui ne vise qu'à donner un premier aperçu de nos observations sur le terrain, concerne principalement le rôle actif et créatif des adolescents dans leur utilisation des médias. Ils regardent certains programmes parce qu'ils les trouvent intéressants, d'autres parce qu'ils sont distrayants, et d'autres parce que « tellement c'est con, ça fait marrer... ». Ils discutent de ces programmes, jouent avec ceux-ci et rejouent des scènes. Il y a des programmes dont on peut s'affirmer spectateur habituel, d'autres dont il ne vaut mieux pas, d'autres encore dont on peut mais en prenant distance. Certains membres du groupe peuvent tout dire, d'autres préfèrent ne pas trop en dire.

Tous ont par ailleurs une connaissance assez réaliste de la production des médias qu'ils regardent, entre autres parce qu'ils aiment regarder des émissions ou lire des articles de presse ou d'internet leur présentant l' " envers du décor » : making-of où on voit comment sont tournées les séries, les émissions, interviews des acteurs, présentateurs, etc.

C'est donc d'adolescents actifs dans leurs multiples utilisations des médias qu'il s'agit, capables de créativité, de prise de distance, de critiques. Des adolescents réflexifs capables de s'expliquer, d'expliquer le sens de leurs différentes pratiques médiatiques. Et finalement des adolescents que l'on ne peut réduire à une seule manière d'utiliser les médias, capables de les adopter toutes simultanément et de les adapter à la situation.

En somme, tout se passe comme si l'environnement médiatique dans lequel ces adolescents évoluent depuis leur naissance s'intégrait pleinement dans leur travail de construction identitaire. Quelles compétences cela suppose-t-il ? C'est précisément ce que nous avons tenté de dégager. 


\section{Compétences médiatiques et construction de l'identité}

La complexité de l'intervention des médias à ce niveau, complexité due principalement au rôle actif des individus, amène certaines difficultés au niveau de la présentation des résultats. Les médias n'interviennent pas de manière unique et unilatérale, nous avons plutôt affaire à une multitude de causes et d'effets inter-reliés. Nous avons néanmoins tenté d'isoler trois compétences développées par les adolescents (l'identification, l'affirmation et la mise en scène), tout en sachant bien que cela n'est qu'une construction théorique visant à faciliter la présentation, et qu'en réalité, ces trois compétences sont fortement interdépendantes. Mais cette complexité est également due à notre volonté de ne pas considérer l'identité comme une boîte noire et de plutôt pénétrer celle-ci, et observer la manière dont les individus gèrent les différentes instances qui la composent, selon notre cadre théorique (identité engagée, identité imaginée, identité assignée). Et à ce niveau, les médias n'interviennent jamais uniquement par rapport à une seule de ces instances, ils interviennent sur plusieurs d'entre elles ; c'est alors le travail de l'individu par rapport à cela qui nous intéresse.

\section{L'identification}

Il est relativement évident que les médias sont porteurs de cultures, de modes de vie, de valeurs morales, de modèles, et pourtant, en affirmant cela, on ne dit rien, on n'explique rien. L'intérêt est de voir ce qu'en font les adolescents.

Ce sont majoritairement les filles qui se sont exprimées dans les groupes et dans les interviews individuelles. Les personnages dont elles nous ont spontanément parlé étaient féminins. On a pu remarquer également que ces peronnages étaient représentatifs de l'état actuel du féminisme (" Ally McBeal », " Bridget Jones's Diary ») et de l'élargissement de la période adolescente, que cet élargissement se fasse dans un sens ou dans l'autre, c'est-à-dire par l'entrée plus précoce dans l'adolescence ( « Dawson's Creek »), ou par le passage plus tardif à ce qui est défini comme l'âge adulte («Friends »). Ce ne sont pas des modèles proprement médiatiques, mais plutôt des modèles de société repris par les médias. 
En ce qui nous concerne, c'est-à-dire au niveau du travail du sujet, le point central est que ces modèles seront retravaillés au sein du groupe de pairs. Effectivement, les personnages dans lesquels l'adolescent peut se retrouver sont des personnages dont il va attendre une meilleure socialisation. L'adolescent peut regarder seul une fiction, mais le groupe est toujours présent à l'intérieur de lui. Quand il retrouvera ses camarades, il en parlera et jouera à imiter tel ou tel personnage (Tisseron, 2000b). Les adolescents se servent donc de certains modèles pour resserrer leur lien avec le groupe, pour trouver une place dans celui-ci.

Les séries valorisées dans ces groupes présentent elles-mêmes des groupes d'amis dans un cadre scolaire, professionnel ou privé. Dans ces groupes fictifs, chaque personnage a une place particulière, un rôle ; en un sens, il représente un archétype : le comique, le stressé, le râleur, etc. Et l'ensemble de ces archétypes semble faire fonctionner parfaitement le groupe, comme si chaque personnage était essentiel. A partir du moment où un groupe réel se reconnaît dans un groupe fictif, et que donc ce groupe fictif est valorisé, imiter un des membres du groupe fictif peut devenir un moyen d'intégrer le groupe réel.

Cela rejoint tout à fait les hypothèses de Serge Tisseron selon lesquelles les phénomènes de groupes ont une importance capitale dans le succès des identifications. Lorsqu'un adolescent choisit de se comporter comme un personnage fictif, que ce soit au niveau du langage, de l'attitude ou de la tenue vestimentaire, c'est toujours par rapport à l'importance que cela lui donne dans son groupe de rattachement. Selon Serge Tisseron (2000a), le phénomène de rattachement à un héros est donc considérablement amplifié par les modes : lorsque la majorité du groupe fonctionne autour d'un héros, les autres suivent parce qu'ils craignent d'être rejetés. La plupart des références ont pour but de socialiser l'individu dans son groupe de référence.

Dans un des groupes rencontrés, on ne pouvait pas réellement parler de « héros ", c'était plutôt un style qui est valorisé : l'humour. C'est donc tous les personnages fictifs ou réels se caractérisant par leur humour qui y sont valorisés. L'exemple le plus frappant concernait, en ce sens, l'humoriste Jamel Debbouze. Lors des interviews de groupe, tout le monde disait l'apprécier et l'imiter de temps à autre, mais tout le monde reconnaissait en Emeline, l'une des filles du groupe, la meilleure imitatrice et la plus grande «fan ». Ce n'est d'ailleurs pas elle qui a répondu, mais les autres qui l'ont présentée comme cela : 
Chercheur : Vous êtes « fans » de quelqu'un?

Marie : Jamel (pour Emeline)!

Chercheur : Tu es « fan » de Jamel ?

Emeline : Euh... oui...

TOUT LE MONDE : Oui, elle l'est !

Chercheur : Et comment on reconnaît quelqu'un qui est «fan »?

Marie : Ben, elle emploie des expressions... Ça revient tout le temps, dès qu'on parle de quelque chose...

C'est donc au niveau du langage que l'imitation est ici apparente. Emeline parle « comme Jamel », ce qui est différent de parler « de Jamel », comme nous le verrons plus loin, bien que l'effet escompté soit le même : se créer une place dans le groupe. L'imitation se fait par rapport à l'importance que cela donne dans le groupe. Et de l'importance, Emeline en a beaucoup, elle est l'un des deux leaders de ce groupe avec Marie. L'adolescent, toujours selon Serge Tisseron, étant en recherche d'intégration, sa quête le portera à élire des héros dans lesquels son groupe peut se reconnaître.

Concernant les différentes instances de l'identité, nous nous trouvons ici au niveau de l'identité assignée : l'individu se fait une idée de ce que les autres attendent de lui, c'est ce qu'il croit devoir faire pour obtenir des autres la reconnaissance à laquelle il aspire. Mais les médias peuvent également intervenir au niveau de son identité imaginée, de ce qu'il voudrait être ou devenir, ou de ce qu'il devrait faire pour assurer son épanouissement, son accomplissement personnel, sa réalisation de soi. Dans ce cas, nous nous trouvons à un tout autre niveau, tant dans le temps que dans l'espace. Ces identifications ne se situent plus maintenant dans le groupe, mais plus tard dans la société. Ce n'est pas ce qu'ils doivent faire maintenant pour être dans le groupe, mais ce qu'ils aimeraient être plus tard pour s'épanouir dans la société. Ces modèles sont exprimés en entretien individuel ou à deux et sont de réels projets de vie future.

Dans les entretiens individuels, le personnage d'Ally McBeal devient ainsi réellement un modèle identificatoire. Caroline et Adline, voulant toutes deux devenir avocates, nous ont expliqué comment elles s'imaginaient à 30 ans : 
Adline : J'espère comme dans «Ally McBeal», parce que elle, elle est avocate, mais elle est quand même...

Caroline : Ils sont en groupe... ça c'est vraiment un truc que j'aime bien...

Adline : c'est vraiment nous...

Caroline : Elle fait son travail...

Adline : Et elle rigole bien à côté...

Caroline : Elle est un peu exaltée mais bon... à côté elle a sa petite vie et elle se marre bien...

Chercheur : Et qu'est-ce qui est intéressant dans cette vie ?

Adline : Ben, c'est qu'elle a vraiment deux personnalités, parce que bon quand elle est avocate, elle doit quand même être sérieuse et tout et en dehors de ça, elle est vraiment tout le contraire... elle est... exaltée...

L'élément qui les intéresse dans le personnage d'Ally McBeal est donc sa manière de concilier le sérieux professionnel et l'amusement privé. Selon Serge Mertens du Centre de Guidance, ce type de modèle joue un rôle rassurant :

Parce qu'ils peuvent avoir l'angoisse de se dire : Mais moi je veux bien rentrer dans le monde des adultes, mais je veux rester celui que je suis. Comment rester fidèle à celui que je suis, avec ma flamme, avec ma force de vie, avec mes envies ? [...] Par contre de voir que quelqu'un peut avoir sa vie professionnelle, déconner, avoir des soucis, s'amuser, tout en travaillant, et qu'on peut être à la fois encore un enfant en rigolant, en s'amusant, je pense que ça les rassure beaucoup. (Serge Mertens, communication personnelle, 14 novembre 2001).

Ce point est particulièrement intéressant en ce qui concerne la construction identitaire, car cela révèle l'importance de la cohérence et de l'unité dans l'identité. Selon Pierre Tap (1979), l'identité personnelle concerne, dans un sens restreint, le sentiment d'identité, c'est-à-dire le fait que l'individu se perçoive le même, identique à lui-même dans le temps. Cet élément est également central dans la définition du sentiment d'identité d'Erikson (1968, p. 14) : « un sentiment subjectif et tonique d'une unité personnelle et d'une continuité temporelle ».

Par rapport à nos interviews, nous pouvons effectivement remarquer qu'Ally McBeal est décrite d'une part comme une avocate et d'autre part, comme une " exaltée ", par Adline et Caroline. Lors d'autres entretiens, elles ont par ailleurs fait part de leur projet de se 
lancer dans des études de droit et se sont définies elles-mêmes comme " exaltées ». Prendre comme modèle Ally McBeal permet alors de projeter une image d'elles-mêmes dans le futur, cohérente avec leur identité actuelle. Il s'agira donc pour elles, tout comme pour Ally, de garder cette constante, cette exaltation, malgré leur emploi d'avocate.

Nous voyons ici le travail du sujet pour gérer les tensions existentielles entre ce que les institutions (médiatiques ou pas) lui ont donné envie de devenir (son identité imaginée), ce qu'elles lui assignent comme limites ou conditions (identité assignée) et ce qu'il a pris dans le passé comme engagement envers lui-même (identité engagée). Elles devront donc parvenir à être avocates, avec l'attitude que cela implique, tout en restant elles-mêmes. Ally McBeal montre que cela est possible et devient en ce sens un modèle auquel s'identifier.

\section{L'affirmation de soi}

La deuxième compétence concerne les phénomènes d'affirmation de soi à partir des préférences et des goûts médiatiques. Du " parler comme », on passe dès lors au «parler de ». Parler d'un personnage ou d'un programme spécifique au sein du groupe, n'est effectivement pas anodin dans la construction identitaire. Cela joue un rôle fondamental. Dominique Pasquier (1999) avait remarqué que les enfants sont bien conscients que ce qu'ils disent de la télévision, ne fût-ce que de dire qu'ils regardent ou non un programme, engage toute leur personne socialement. Ils apprennent donc à opérer le travail de figuration nécessaire pour entrer en conformité avec les normes et valeurs des groupes dans lesquels ils cherchent à s'insérer. « Ils apprennent à nier certains goûts, à refouler des préférences, ou au contraire à regarder pour entrer dans une communauté de téléspectateurs. » (Pasquier, 1999, p.21). En ce sens, parler d'un personnage permet de parler de soi, de s'affirmer : la déclaration des choix et des préférences est un message personnel destiné à autrui sous couvert d'un personnage.

Il semble effectivement qu'il y ait une correspondance entre la justification du choix d'un programme et la présentation de soi. Lors des premiers entretiens, nous demandions aux jeunes quels étaient leurs programmes (films, séries, émissions) préférés et d'expliquer pourquoi. Après plusieurs entretiens, quand ils étaient seuls et plus en confiance, nous leur demandions de se présenter, de dire qui ils étaient. La similitude des réponses à ces deux questions fut particulièrement éclairante. 
Mélanie : Moi, un film que j'adore, c'est «Danse avec les Loups » et pourquoi... je pense que c'est parce que j'adore regarder les paysages et les musiques dans les films et y a vraiment des trucs très beaux là-dedans... et je suis très sensible donc...

Et, plus tard, lorsqu'elle se définit elle-même :

Mélanie : Moi, je crois que je dirais que je suis une personne très sensible et que je m'émerveille des choses les plus petites qu'il y a dans la vie.

N'étant pas très à l'aise dans le groupe face aux leaders, elle n'avait presque pas parlé lors des entretiens de groupe, et n'avait pas participé à la discussion sur les films préférés. Elle nous explique alors pourquoi elle ne l'a pas fait :

Mélanie : Non, en groupe, moi, je n'aurais pas dit que j'étais une personne très sensible et tout... parce que c'est quelque chose qui est vu de manière nunuche.

On comprend maintenant pourquoi le fait de citer tel ou tel programme peut être " dangereux » pour l'image que les autres auront de soi : en citant un programme, l'individu s'affirme, c'est de lui dont il parle également. Suivant la place que l'on a dans le groupe, suivant la reconnaissance qu'a ce programme dans le groupe, il y a donc des choses que l'on peut dire et d'autres qu'il vaut mieux cacher.

En ce sens, le fait que les sujets soient prêts à nier ou changer leurs préférences médiatiques, du moins en public, vient conforter notre idée d'une implication de l'identité entière du sujet dans l'affirmation de ses choix médiatiques.

Et c'est exactement la même chose qui se passe avec le choix d'un personnage. Les adolescents projettent certains de leurs traits identitaires sur le personnage fictif ; ce faisant, quand ils disent que ce personnage leur ressemble, ils parlent en réalité d'eux-mêmes. La plupart du temps, lorsqu'ils expliquent pourquoi tel ou tel personnage est leur personnage préféré, ils expliquent qu'il est « comme eux ». Pour Serge Mertens, les médias sont un lieu de projection inconscient ; quand les adolescents citent une vedette, c'est ce qu'ils supposent de cette vedette, ce qu'ils projettent sur cette vedette, et qu'ils découvrent quelque part finalement, par ce support projectif, de leur propre identité. Il y a donc un effet retour : par la projection, ils se découvrent eux-mêmes. 
Mais en tant que références communes, partagées par tous, les programmes médiatiques permettent également de socialiser les émotions. Serge Tisseron (2000b) donne ainsi cet exemple à propos des images violentes : racontez vos malheurs familiaux à vos amis, ils ne vous écouteront pas ; racontez la dernière séquence d'un film violent que vous avez vu, ils vous écouteront. Il nous semble que cette pratique ne se limite pas uniquement aux images violentes, mais à toutes les images, et à plus forte raison si elles renvoient, chez l'individu particulier, à certaines émotions. Nous avons effectivement pu observer qu'une fiction médiatique pouvait être le point de départ d'une discussion sur des problèmes réels. Deux exemples sont revenus plusieurs fois : le divorce des parents et les ruptures amoureuses.

Mélanie : Par exemple, si dans un film, on peut voir quelqu'un qui trompe sa copine avec une autre et puis que l'autre l'apprend ou des trucs dans le genre... ça se passe vraiment à l'école parfois et ça alimente parfois les sujets de conversation...

Chercheur : C'est ce qui se passe dans la série ou dans la réalité qui alimente les conversations?

Mélanie : Mais on parle de la série parfois pour arriver dans les vrais sujets de conversation par rapport à ce qu'il se passe à l'école.

Mélanie : [...] comparer ce qu'il se passe dans les deux... parce que moi aussi mes parents sont divorcés, et quand je vois des films comme ça, parfois je me dis : "Tiens, il vit ça comme ça... et moi, je l'ai plutôt vécu comme ça... ».

Comme on le voit, les médias permettent donc d'une part de s'affirmer, dans le sens où parler d'un film, c'est parler de soi ; et d'autre part, de socialiser ses émotions par le détour en images. Le premier phénomène a été observé par la correspondance quasi-systématique entre les justifications du choix d'un programme ou personnage préféré et la manière dont ils se présentent; et le deuxième par le fait qu'ils font abondamment référence aux fictions pour ensuite parler de leurs problèmes. 


\section{La mise en scène de soi et d'autrui}

Ce que nous appelons « mise en scène de soi » est le processus par lequel l'individu va expérimenter des rôles, des comportements et des attitudes, d'une part en se projetant dans des situations fictives et d'autre part, en ramenant ces situations dans sa vie réelle. La « mise en scène d'autrui » est, quant à elle, le processus par lequel l'individu projette autrui dans une situation fictive, donnant ainsi une certaine image de cet autrui. Par ce jeu, l'individu va donc expérimenter des traits identitaires et éprouver l'image que les autres ont de lui. De même, en l'imaginant dans telle ou telle situation, autrui lui propose la manière dont il le perçoit. Par cet aller-retour entre la fiction et la réalité, on expérimente des traits identitaires et on teste la reconnaissance de ceux-ci au sein du groupe de pairs. Le terme de reconnaissance doit ici être compris dans le sens d'une recherche de " consonance » entre l'image que le sujet présente de lui-même et l'image qu'autrui lui renvoie.

Le groupe est davantage, ici, un « laboratoire relationnel », un espace de relations au sein duquel, l'adolescent peut expérimenter sa propre identité et la reconnaissance de celle-ci.

En ce qui concerne les médias, c'est peut-être à ce niveau qu'ils sont le plus utilisés. Chaque jour, les adolescents sont confrontés dans les fictions (films et séries), mais également dans les émissions comme les reality-shows, à une multitude de rôles, de traits identitaires et de manières de réagir qu'ils pourront ensuite expérimenter dans leurs relations sociales.

Et concernant la construction identitaire, observer « la mise en scène de soi » permet de rentrer pleinement dans le travail du sujet pour construire son identité personnelle. Se faisant une idée de ce qu'il est, de ce que les autres attendent de lui, tout en restant en accord avec ses engagements, l'adolescent pourra construire un personnage qu'il mettra en scène dans la dramatique de sa quête de reconnaissance.

Il nous semble qu'à ce niveau, les médias jouent un rôle important, l'image de l'autre est bien souvent proposée à l'aide d'autres images, reprises des fictions médiatiques, en mettant en scène autrui. Au lieu de verbaliser l'image que l'on a d'autrui, on va plutôt faire référence à un personnage ou une situation. Ainsi, il semble plus facile - ou plus complet - de dire à autrui : « toi, je t'imagine bien réagir comme un tel dans la série !», que de se lancer dans une description détaillée de la personnalité d'autrui. Bien évidemment, cette image a une fonction ambivalente : elle peut aider à la découverte de soi et confirmer le sujet 
dans son identité ; mais elle peut aussi, selon Lipiansky (1992), l'aliéner, à travers son désir de correspondre à l'image que l'autre attend de lui.

Marie : On compare les gens : le susceptible du groupe, on le compare à un tel...Un peu comme la «top-biche » du groupe, ce sera Emeline !

Emeline : Ou alors Andréa...[à] Phoebe ${ }^{1}$.

Marie : Oui, voilà !

Emeline : La fille qui ne sait jamais rien...

Marie : Elle ne sait pas de quoi on parle, elle s'amène et elle dit n'importe quoi!

Ce que l'on peut observer, c'est donc que l'adolescent utilise abondamment les images médiatiques pour faire part à autrui de la manière dont il le perçoit. Et il est particulièrement intéressant de remarquer que ce processus se fait tout naturellement, tout simplement au détour d'une phrase. Autant quand on leur demande de se décrire l'un l'autre avec des mots, l'exercice semble compliqué et gênant ; autant lorsqu'ils passent par les images, donner son avis sur autrui se fait avec une facilité déconcertante. Nos entretiens regorgent d'exemples de ce type : lorsque l'on analyse attentivement leurs discussions, on voit apparaître une multitude de petites phrases, souvent exprimées avec des éclats de rire, entre deux phrases plus sérieuses, où une image d'autrui est proposée. Et lors des entretiens par deux, quand nous leur demandions de se décrire l'un l'autre avec des mots cette fois-ci, ce qui était dit correspondait aux images médiatiques exprimées lors des discussions de groupe. Il est important de souligner cette correspondance afin de remarquer que les images et les mises en scène d'autrui correspondent réellement à la perception que l'individu se fait d'autrui et n'ont pas uniquement pour but de provoquer le rire ou la moquerie.

La « mise en scène d'autrui » permet donc de donner rapidement l'image que l'on a d'autrui, en dédramatisant cet exercice difficile et gênant consistant à avouer à autrui la manière dont on le perçoit. Cela ressemble davantage à un jeu auquel chacun participe honnêtement. Mais cette honnêteté reste sur le mode ludique, et en ce sens, le processus est facilité.

1 Personnage de la série « Friends ». 


\section{Conclusion}

Cette présentation était bien évidemment trop brève pour rendre compte de toute la complexité des modalités d'intervention des médias dans la construction identitaire des adolescents et des longues heures d'entretiens que nous avons eues avec eux. Nous pouvons néanmoins dégager trois compétences qui, elles-mêmes, peuvent prendre deux dimensions.

L'identification renvoie aux pratiques du " parler / agir comme... » et se compose de deux sous-modalités : l'imitation et la projection. L'adolescent imite certains traits identitaires, repris chez un personnage fictif valorisé dans le groupe, en vue de resserrer ses liens avec ce groupe d'appartenance, ici et maintenant. Et à plus long terme, l'adolescent se projette dans des modèles d'épanouissement personnel, en cohérence avec son identité actuelle.

L'affirmation de soi renvoie aux discussions se rapportant aux médias et donc au " parler de... » et comprend la justification des préférences et la socialisation des émotions. Les discussions autour de leurs personnages, séries ou films préférés sont autant d'occasions et de possibilités de parler de soi, de s'affirmer. Il y a toujours une grande correspondance entre la justification des préférences et la présentation de soi. Mais les discussions à partir des fictions médiatiques sont également un moyen de socialiser des faits qui relèvent du privé.

La mise en scène renvoie aux jeux d'imagination et de répétitions de scènes reprises des fictions médiatiques, c'est-à-dire au « faire comme si » et se compose également de deux sous-modalités : l'expérimentation des comportements et la recherche de consonance. En répétant certaines scènes, l'adolescent a la possibilité, au sein de son groupe d'appartenance d'expérimenter différents comportements, que ceux-ci renvoient à des rôles sociaux ou à de simples traits identitaires. Et cette mise en scène de soi et d'autrui est également un moyen, pour l'adolescent, de confronter l'image qu'il a de lui-même avec la manière dont autrui le perçoit. Dans cette recherche de consonance, les médias constituent une source inépuisable de références imagées.

Ces différentes compétences, dégagées dans cette recherche, montrent donc d'une part le rôle actif de ces spectateurs dans leur rela- 
tion aux médias et, d'autre part, l'importance du groupe de pairs dans cette utilisation des contenus médiatiques.

\section{Références}

Bajoit, G. (1997). Qu'est-ce que le sujet? Dans G. Bajoit \& E. Belin(Ed.), Contributions à une sociologie du sujet. (pp. 113-130). Paris : L'harmattan.

Dayan, D. (1992). Les mystères de la réception, Le Débat, 71, 146-162.

Erikson, E.H. (1968). Adolescence et crise. La quête de l'identité. Paris : Flammarion.

Katz, E., \& Liebes, T. (1990). The export of meaning : cross cultural readings of Dallas. New-York : Oxford University Press.

Liebes, T. (1994). A propos de la participation du téléspectateur, Réseaux, 64, 93-104.

Lipiansky, E.M. (1992). Identité et communication. Paris : PUF.

Pasquier, D. (1994). Hélène et les garçons : une éducation sentimentale, Esprit, 6, 125145.

Pasquier D. (1995). Chère Hélène. Les usages sociaux des séries collège, Réseaux, 70, 9-39.

Pasquier, D. (1999). La culture des sentiments. L'expérience télévisuelle des adolescents. Paris : Editions de la Maison des sciences de l'homme.

Tap, P. (1979). Identité individuelle et personnalisation, colloque international Toulouse-septembre, Paris, Sciences de l'homme.

Tisseron, S. (2000a). Enfants sous influence. Les écrans rendent-ils les jeunes violents? Paris: Armand Colin.

Tisseron, S. (2000b). Le public jeunesse face aux séries télévisées. Communication au colloque « Jeunesse et Médias » organisé par le Conservatoire européen d'écriture audiovisuelle (CEEA), Paris, 13 et 14 mars 2000. 